\title{
Sistema integrado para empresas de construcción en Cusco
}

\author{
Alicia CubA ViLlenA \\ Universidad Europea de Energía y Medio Ambiente (UEMA) \\ aliciacubal@gmail.com
}

Recibido: 23 de noviembre del 2015

Enviado a evaluar: 23 de noviembre del 2015

Aceptado: 30 de noviembre del 2015

\section{RESUMEN}

Actualmente las empresas requieren estrategias innovadoras, que permitan gestionar de forma integral, optimizando los recursos y maximizando los resultados. Siendo el objetivo del presente trabajo de investigación, diseñar un instrumento de gestión integral (gestión de la calidad, ambiental, seguridad y salud ocupacional) para el sector construcción en Cusco. Se realizó un análisis actual de la actividad de construcción, se planteó a diferencia de otras propuestas, el diseño que inicia con el diagnóstico, planificación, organización, ejecución, supervisión y optimización del sistema integral. Los resultados están expresados en el diagnóstico de los 3 sistemas, información clave, para el planteamiento y propuesta de las etapas posteriores; estructurando el planteamiento de los subprogramas basados en el diagnóstico integral, para finalmente determinar los lineamientos estratégicos, de implementación, evaluación y verificación del sistema, teniendo en cuenta la norma ISO 9001: 2008, ISO 14001:2004 y OSHAS 18001:2007; así como la legislación vigente para el Perú.

Palabras clave: Sistema, gestión integral, construcción, Cusco.

\section{Integrated system for construction companies in Cusco}

\begin{abstract}
Currently companies require innovative strategies, to manage in a comprehensive way, optimizing resources and maximizing results. Being the objective of this research work, designing an instrument of integral management (quality management, environmental, occupational health and safety) for the construction sector in Cusco. There was a current analysis of construction activity, was raised in contrast to other proposals, the design that starts with diagnosis, planning, organization, implementation, monitoring and optimization of the integrated system. The results are expressed in the diagnosis of the 3 systems, key information, for the approach and proposal of the later stages; structuring approach of subprogrammes based on a comprehensive diagnosis, to finally determine the strategic guidelines, implementation, evaluation and verification of the system, taking into account the ISO 9001: 2008, ISO 14001:2004 and OHSAS 18001:2007; as well as the current legislation for Peru.
\end{abstract}

Key words: System, integral management, construction, Cusco. 


\section{Entreprises de construction intégrés dans le Système Cusco}

\section{RÉSUMÉ}

Actuellement les sociétés exigent des stratégies novatrices, afin de gérer de façon globale, optimiser les ressources et maximiser les résultats. Étant l'objectif de ce travail de recherche, concevoir un instrument de gestion intégrale (gestion de la qualité, de l'environnement, santé et sécurité au travail) pour le secteur de la construction dans Cusco. Il y avait une analyse courante de l'activité de construction, a été soulevée en contraste avec d'autres propositions, la conception qui commence avec le diagnostic, la planification, organisation, mise en oeuvre, le suivi et l'optimisation du système intégré. Les résultats sont exprimés dans le diagnostic des 3 systèmes, information clé, pour l'approche et la proposition de les étapes ultérieures; approche de structuration des sous-programmes fondés sur un diagnostic approfondi, pour finalement déterminer les orientations stratégiques, la mise en oeuvre, l'évaluation et la vérification du système, en tenant compte de l'ISO 9001: 2008, ISO 14001:2004 et OHSAS 18001:2007; ainsi que la législation actuelle pour le Pérou.

Mots clés: Système, gestion intégrée, la construction, Cusco.

\section{INTRODUCCIÓN}

En el contexto actual de gestión de las entidades públicas y privadas, aparecen una serie de exigencias de orden legal, de los clientes o del mercado, que obligan a estas organizaciones a implementar sistemas de gestión organizacional como herramientas gerenciales de mejoramiento, por esta razón y bajo el principio de "una sola gestión, un solo equipo", se presenta una propuesta de sistema de gestión integral SGI, que facilite el abordaje de todas las exigencias normativas orientadas al propósito básico de mejorar la gestión y los resultados de la entidad que lo aplica. (Hurtado, F. A. A., 2008). El nuevo entorno económico competitivo que surge en la década de los noventa, cuyas características son la globalización y la transformación de las economías industriales, impuso a las empresas y a sus administradores afrontar nuevos desafíos. Estos sistemas se han visto obligados a evolucionar drásticamente en los últimos tiempos, para adaptarse a los diferentes, rápidos e importantes avances, tanto tecnológicos, en el mundo empresarial en los últimos años. (Oltra Badenes, R. F., 2012). Actualmente la integración de los sistemas de gestión es una realidad que las organizaciones comienzan a ver como un forma de optimizar sus procesos como un visión multidisciplinar para potenciar sus resultados y garantizar su viabilidad en el mercado a mediano y largo plazo (Pardo, C. I., 2008). El propósito de un sistema integrado de gestión es ayudar a proporcionar una clara representación de todas las funciones de su respectivo sistema de gestión, para mostrar cómo impactan y complementan unos a otros y demostrar que su relación le ayuda en la gestión de los respectivos sistemas de gestión riesgos de la organización. Por supuesto, uno de los objetivos principales es el de ayudar a asegurar la sinergia y para reducir la duplicación y racionalización de los enfoques comunes, ideas y herramientas. (Pardy, W., 2009). Los sistemas de gestión por separado, tratan procesos internos relacionados con áreas determinadas, como calidad, medio ambiente, seguridad y 
salud laboral y otras. Con un sistema integrado de gestión se puede adoptar una visión global que permita mejorar los procesos internos y evaluar todos los sistemas en una sola auditoría de certificación. (Pastor, J. R., 2006). El sistema de gestión es una herramienta que permite la sistematización, contribuyendo a optimizarla, se puede dividir en 2 fases: la planificación estratégica y la implementación estratégica, la primera relacionada con la previsión de las actividades, restablecimiento de objetivos a través de la selección de una estrategia adecuada. Y la segunda concierne a la toma de decisiones, del desarrollo de la estructura organizativa, cumplimiento con efectividad de las mismas, y control de la eficacia de las mismas. (Sánchez, C. E. A., 2006). Existen actualmente varios modelos de gestión que las empresas están adoptando como modelo para que sus organizaciones se encaminen hacia lo que se ha dado en llamar excelencia. Por un lado las normas ISO 9000 de calidad son un punto ineludible de referencia, como también lo son las normas ISO 14000 de gestión ambiental, desarrolladas a semejanza de las primeras. En el nuevo marco reglamentario sobre prevención de riesgos laborales, inspirado en principios básicos, como la mejora continua y la integración de la acción preventiva en la actividad de la empresa, es obviamente no solo una exigencia, sino también una necesidad para dar respuesta a los requerimientos que la persona tiene en su ámbito laboral, garantizándole unas condiciones de trabajo dignas, y potenciando su desarrollo profesional (Santos, J. R. M., 2004). Por lo que el objetivo del presente trabajo es realizar un diseño del sistema integrado de gestión para una empresa de construcción en Cusco, ya que actualmente la industria de construcción en Cusco no cuenta con instrumentos de gestión que le permitan establecer una competitividad empresarial, permitiendo que este trabajo se constituya en un modelo de gestión innovador, estableciendo la eficiencia y eficacia de todos los procesos y actividades que se realizan en la empresa, optimizando todos los recursos existentes en beneficio del sistema proporcionando calidad, seguridad y conservación del ambiente. En el ámbito privado y a iniciativa de las organizaciones internacionales surge el sistema de gestión integral, con el propósito de equilibrar los objetivos de rentabilidad, equidad y de sostenibilidad. La implementación del SIG busca un enfoque integral de los procesos que permitirá no sólo el mejor uso de los recursos y la simplicidad en el manejo de los procesos, y al aumento de la rentabilidad. (Cachay, 2009). Con ello se propone a las organizaciones que muestren su responsabilidad social empresarial (RSE), mediante acciones que demuestre desde el punto de vista empresarial: no contaminación, respeto y preocupación por sus trabajadores, clientes y stakeholders en general. Proporciona, por tanto, a las organizaciones una justificación válida para incursionar en acciones de RSE, que podrían convertirse en empresas competitivas y socialmente responsable con criterios de excelencia. (Quintero G., M. L., 2013). Otra de las tendencias futuras identificadas son el tratamiento especial y herramientas de ayuda a la toma de decisiones particulares y adecuadas tratamiento específico de la información; ello supone una adaptación de estos sistemas integrados de gestión a sectores industriales concretos y a sus requerimientos de negocio específicos. En línea con esta tendencia, se presenta e investiga una problemática concreta, (Oltra Badenes, R. F., 2012). 


\section{MÉTODO}

La metodología usada es de enfoque cualitativo de lógica inductiva: para la elaboración del diseño del sistema integrado de gestión, se realizaran los siguientes procedimientos y formatos específicos:

Etapa I: Diagnostico de la situación actual: Aspectos comunes entre los sistemas de calidad, ambiental y SSO.- Se realizó la descripción de los aspectos comunes entre los sistemas que permitan, integrar los procedimientos y métodos optimizando los recursos. (Fomento, M. D., 2005).

\section{Etapa II diseño de estrategias de mejoras:}

a.- Identificación de los requisitos.- Para el adecuado diseño del sistema integrado de gestión se realizó la identificación de requisitos, realizando una identificación y evaluación previa de los procesos. También obliga a repasar los requisitos legales, para concluir con cuadros y representaciones gráficas de los procesos para los 3 sistemas, incorporando los aspectos de calidad, ambientales y de riesgos - salud ocupacional en cada etapa. (Fomento, M. D., 2005).

B.-Despliegue de los requisitos.- Una vez identificados los procesos y los requisitos hay que circunscribirlos o asignarlos a un método (procedimiento). Un procedimiento refleja las acciones operativas, de supervisión, de comunicación y de registro de datos de un proceso. La evaluación se realizó con la descripción de programa y subprogramas de los requisitos con acciones preventivas y correctivas. (Fomento, M. D., 2005).

C.-Integración de métodos y documentos.- Planteamiento de un solo manual tiene que integrar los elementos comunes e incorporar los elementos diferenciados, para lo cual se deberá realizar una tabla detallada relacionada con los procedimientos del sistema integrado de gestión, (Fomento, M. D., 2005). Describiendo las particularidades de la industria de construcción del Cusco.

\section{RESULTADOS}

\subsection{DIAGNÓSTICO DE CALIDAD, AMBIENTAL, SEGURIDAD Y SALUD OCUPACIONAL DEL SECTOR CONSTRUCCIÓN EN CUSCO}

Según (Sánchez C. E. A. et al., 2006); para la integración de los tres sistemas de gestión se debe seguir el siguiente programa: Análisis de la situación inicial en calidad, seguridad y medio ambiente; evaluación y toma de datos (Fomento, M. D., 2005). b.-adecuación de la legislación vigente y a la normativa de tipo voluntario, c.definición del grado de integración que queremos lograr, d.- establecimiento del programa de tareas a realizar, recursos necesarios, responsables, y plazos, 
implantación del sistema, seguimiento y control, formación y difusión para dar a conocer el sistema y las modificaciones, auditoría de certificación. (Sánchez, C. E. A., 2006).

\subsubsection{EVALUACIÓN E IDENTIFICACIÓN DE LOS ESTÁNDARES DE CALIDAD}

Donde los estándares de calidad producidos en las diferentes etapas de la construcción, fueron identificados y son los siguientes:

Figura 1. Los estándares de calidad de la actividad de construcción.

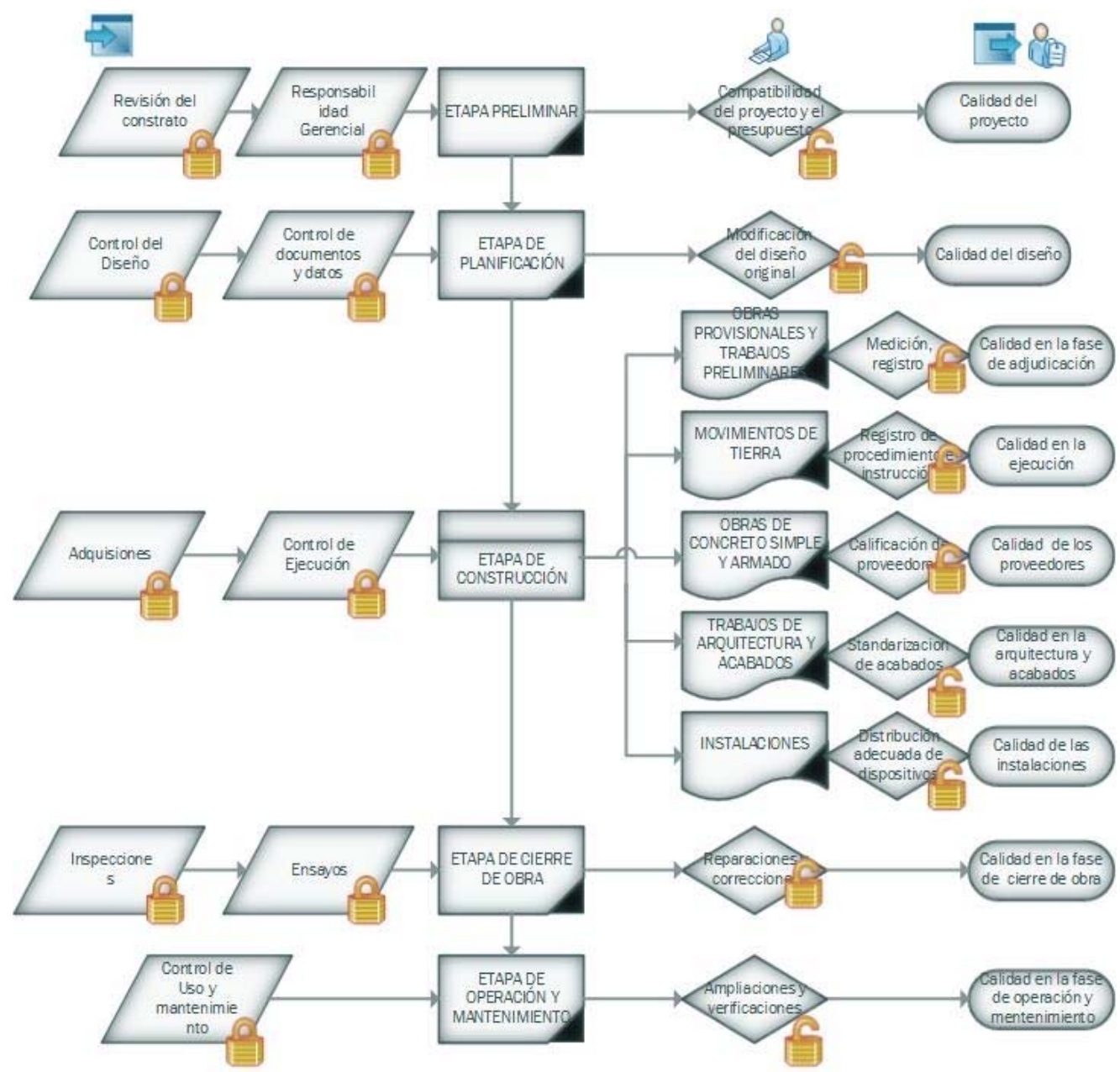

Fuente: Elaboración propia. 
Según la figura 1 se realiza la evaluación del diagnóstico de los estándares de calidad definiéndose varias etapas:

Etapa preliminar: se realizó el análisis de la gestión de la calidad, realizando la revisión del contrato, designando la responsabilidad gerencial para establecer la compatibilidad del proyecto, el presupuesto y mejorar la calidad del proyecto.

Etapa de planificación: Se planificó la gestión de la calidad, a través del control del diseño, control de documentos y datos, que permiten las mejoras y modificación del diseño original, garantizando la calidad del diseño.

\section{Etapa de Construcción, se realizó}

A.- la Implementación y aseguramiento de la gestión de la calidad, con verificación de las adquisiciones, medición y registro, asegurando la calidad en la fase de adjudicación; así como el registro de procedimientos e instrucciones que garantizan la calidad en la ejecución.

B.- Control de ejecución con la calificación de proveedores, que definen la calidad de los proveedores; la estandarización de acabados, que determinan la calidad en la arquitectura y acabados, y la distribución adecuada de dispositivos, que permiten la calidad de las instalaciones.

Etapa de Cierre de obra, donde se realiza el control y verificación de la gestión de la calidad, a través de ensayos e inspecciones, para corregir las reparaciones y correcciones, que garantizan la calidad en la fase de cierre de obra.

Etapa de Operación y mantenimiento, permite el control y verificación de la gestión de la calidad, con el control del uso y mantenimiento, las ampliaciones y verificaciones que aseguran la calidad en la fase de operación y mantenimiento.

\subsubsection{EVALUACIÓN E IDENTIFICACIÓN DE LOS ASPECTOS Y POTENCIALES IMPACTOS AMBIENTALES SIGNIFICATIVOS}

Los aspectos y potenciales impactos ambientales significativos a generarse en las diferentes etapas son los siguientes: 
Figura 2. Aspectos e impactos de los Procesos de la actividad de construcción.

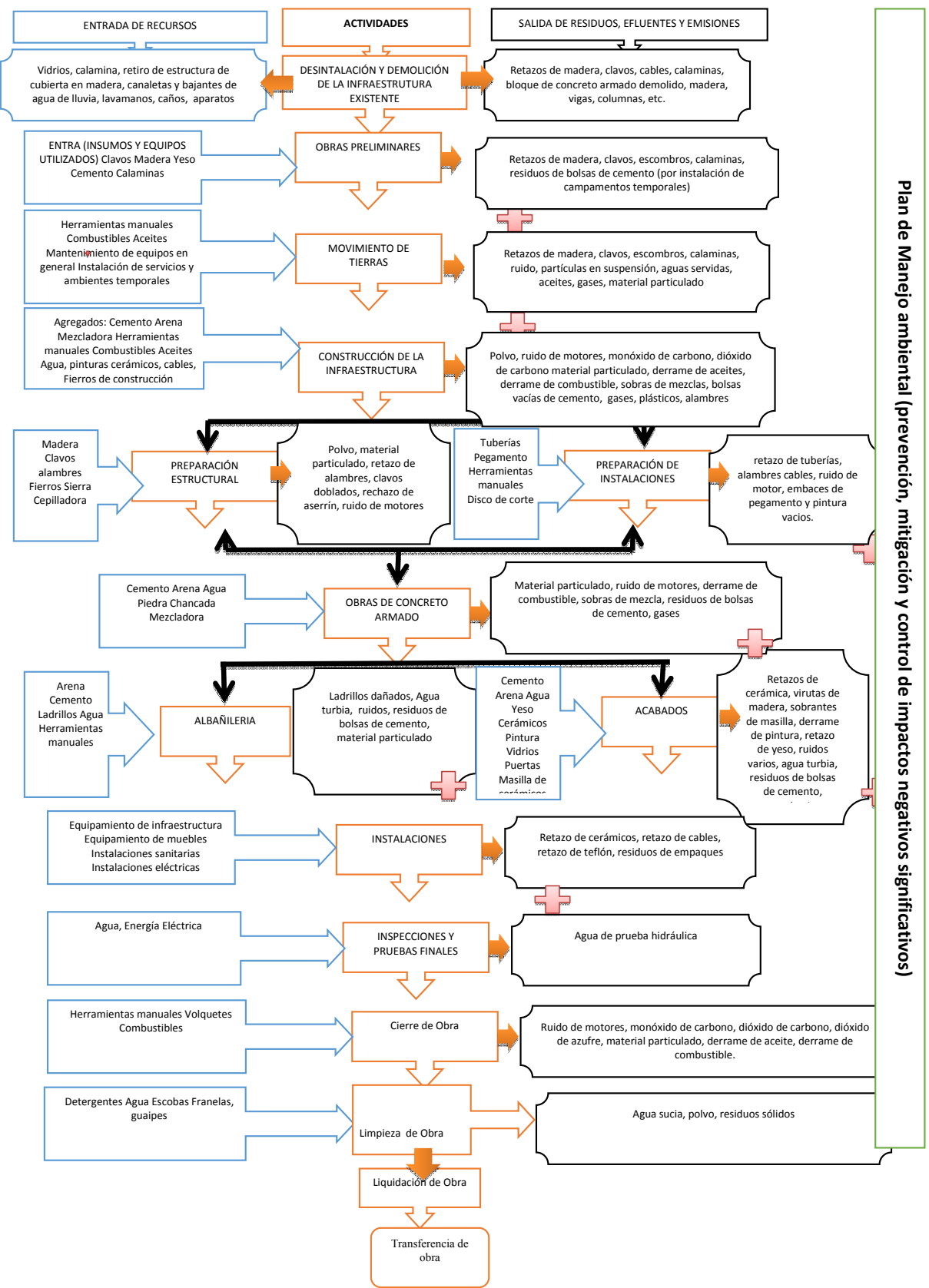

Fuente: Elaboración propia. 
Según la figura 2 se realiza la evaluación del diagnóstico de los aspectos e impactos ambientales definiéndose varias etapas:

Etapa de Construcción, Cierre, Operación y mantenimiento.- se identifican dos matrices afectadas agua y suelo, donde los aspectos ambientales, para la matriz agua son, descarga de aguas servidas (contaminación de cuerpos acuíferos), consumo irracional del recurso hídrico (contaminación del recurso hídrico), para la matriz suelo son descarga de aguas servidas (contaminación y degradación de suelos), derrame de hidrocarburos, aceites y derivados, detergentes (contaminación del manto acuífero), movimiento de tierras (contaminación sonora por la maquinaria usada; incremento de material particulado.), generación de residuos sólidos orgánicos (contaminación y degradación de suelos).

En la etapa de construcción y cierre de obra.- la matriz ambiental afectada es el aire, presentando los siguientes aspectos ambientales, emisión de material particulado por movimiento de tierras (daños a la salud), emisión de gases contaminantes $\mathrm{CO}_{2}$, $\mathrm{CO}$ y $\mathrm{SO}_{2}$ (contaminación del aire), emisiones sonoras de la operación de la maquinaria y equipos durante la construcción (alteración de la fauna existente, contaminación sonora, visual).

\subsubsection{IDENTIFICACIÓN DE PELIGROS Y EVALUACIÓN DE RIESGOS LABORALES}

Según la figura 3 se realiza la evaluación del diagnóstico de la seguridad y la salud ocupacional definiéndose varias etapas:

Etapa preliminar.- Se realizó el análisis de la gestión de (salud, seguridad ocupacional) SSO, con la identificación de peligros y riesgos de SSO y la designación del responsable, que permite la evaluación de riesgos SSO.

Etapa Planificación.- Con la planificación de la gestión de la SSO, se realiza el control del diseño (acciones correctivas y preventivas), control de documentos y datos (identificación de peligros, evaluación y control de riesgos (IPERC)); que establece la modificación de la evaluación de riesgos SSO que garantiza un objetivo plan de prevención y corrección de riesgos SSO.

Etapa Construcción.- Realizando la implementación y aseguramiento de la gestión de la SSO, a través de a.- la matriz de riesgos SSO con un registro y charlas diarias de SSO), la preparación del plan de emergencia y entrenamiento, procedimientos, respuesta y revisión del plan.

Etapa Cierre de obra.- Con el control y verificación de la gestión de la SSO, realizando las inspecciones de SSO y exámenes ocupacionales finales permitiendo la 
revisión de las acciones preventivas y correctivas con la verificación del proceso de SSO del cierre de obra.

Etapa Operación y mantenimiento.- A través del control y verificación de la gestión de la SSO, con la identificación de peligros, evaluación de riesgos de $\mathrm{SSO}$, asegura el plan de Prevención y corrección de riesgos SSO para la infraestructura en operación.

Figura 3. Identificación de peligros y evaluación de riesgos laborales.

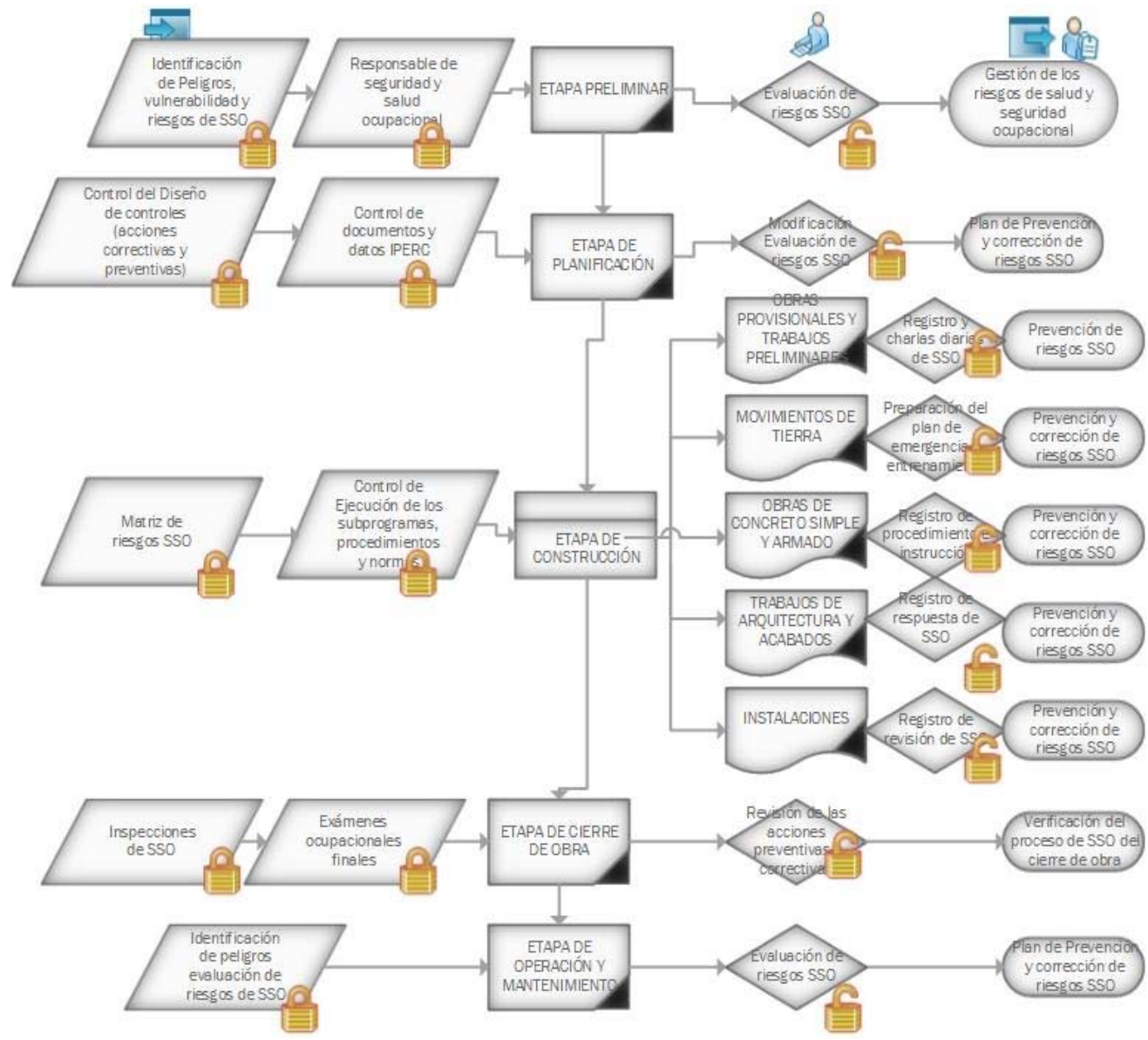

Fuente: Elaboración propia. 
En base al diagnóstico preliminar de los 3 sistemas de la industria de la construcción nos permite la sistematización del sistema de gestión integral para el sector de la construcción. La base para implementar un sistema de gestión integral, será considerar la interconexión e integración de las siguientes áreas: Proceso Estratégicos (Área gerencial); Proceso Operativos (Área técnica, Área de logística); Proceso Soporte: (Área administrativa, Área de personal).

Figura 4. Sistema Integrado de Gestión.

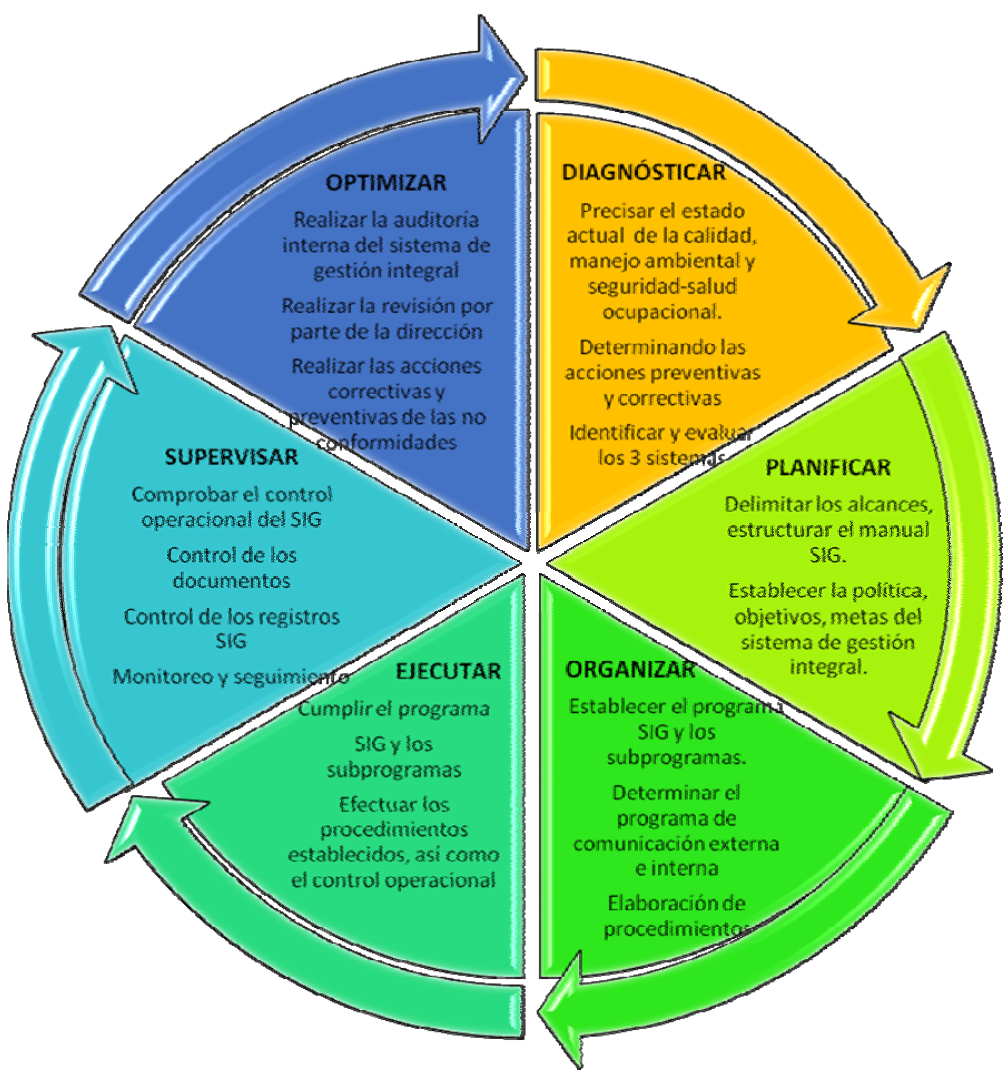

Fuente: Modificación del ciclo de Deming.

En la figura 4 podemos observar, que para plantear el sistema de gestión integral es necesario realizar el diagnóstico y evaluación de cada uno de los sistemas que deseamos integrar, los cuales constituirán el fundamento base para nuestra propuesta final. 
Figura 5. Diseño del Sistema Integrado de Gestión.

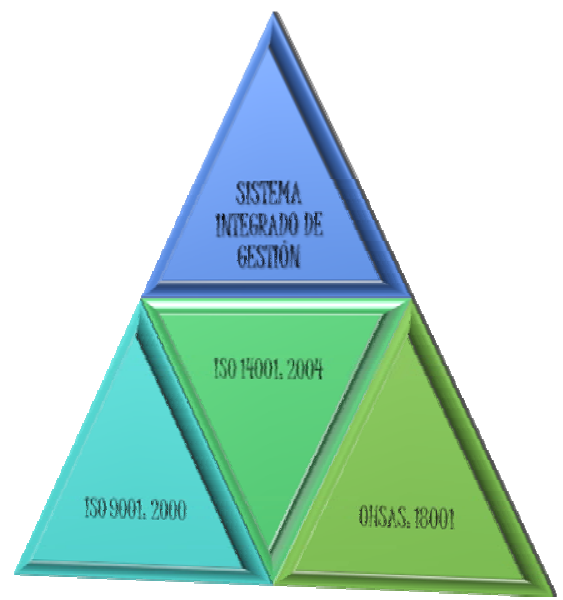

Fuente: Elaboración propia.

En la figura 5, los sistemas de gestión presentados, contienen los requisitos basados en un proceso dinámico de mejora continua, que considera la metodología conocida como ciclo de Deming y Shewart por lo que, en la presente investigación, no se inicia con la planificación según estos autores, sino se mejora este proceso, según lo mencionado (Modificación de Fomento, M. D., 2005), (Sánchez, C. E. A., 2006); "realizar un análisis y diagnóstico de la situación actual" que se concretiza en Diagnosticar, porque para planificar o desarrollar cualquier instrumento de gestión es indispensable el conocimiento del estado actual del mismo, identificando y describiendo la situación de la empresa o institución previa al planteamiento de la propuesta permitiendo describir siguiente: Diagnósticar: Precisando el estado actual de la calidad, manejo ambiental y seguridad-salud ocupacional, determinando las acciones preventivas y correctivas, identificar y evaluar los 3 sistemas, Planificar: Luego del diagnóstico se delimitan los alcances, se estructura el manual SIG, estableciendo la política, objetivos, metas del sistema de gestión integral. Organizar: Después de la planificación se organiza y establece el programa SIG y los subprogramas, determinando el programa de comunicación externa e interna, con la elaboración de procedimientos. Ejecutar: Posterior a la organización se deben realizar el cumplimiento del programa, SIG y los subprograma, efectuar los procedimientos establecidos, así como el control operacional. Supervisar: A continuación se supervisa, comprobando el control operacional del SIG, con el control de los documentos, control de los registros SIG, monitoreo y seguimiento. Optimizar: Finalmente posterior al monitoreo y seguimiento, se identifican los aspectos que permitan optimizar el SIG, realizando la auditoría interna del sistema de gestión integral, realizando la revisión por parte de la dirección, realizando las acciones correctivas y preventivas de las no conformidades. 
Figura 6. Diseño del SIG.

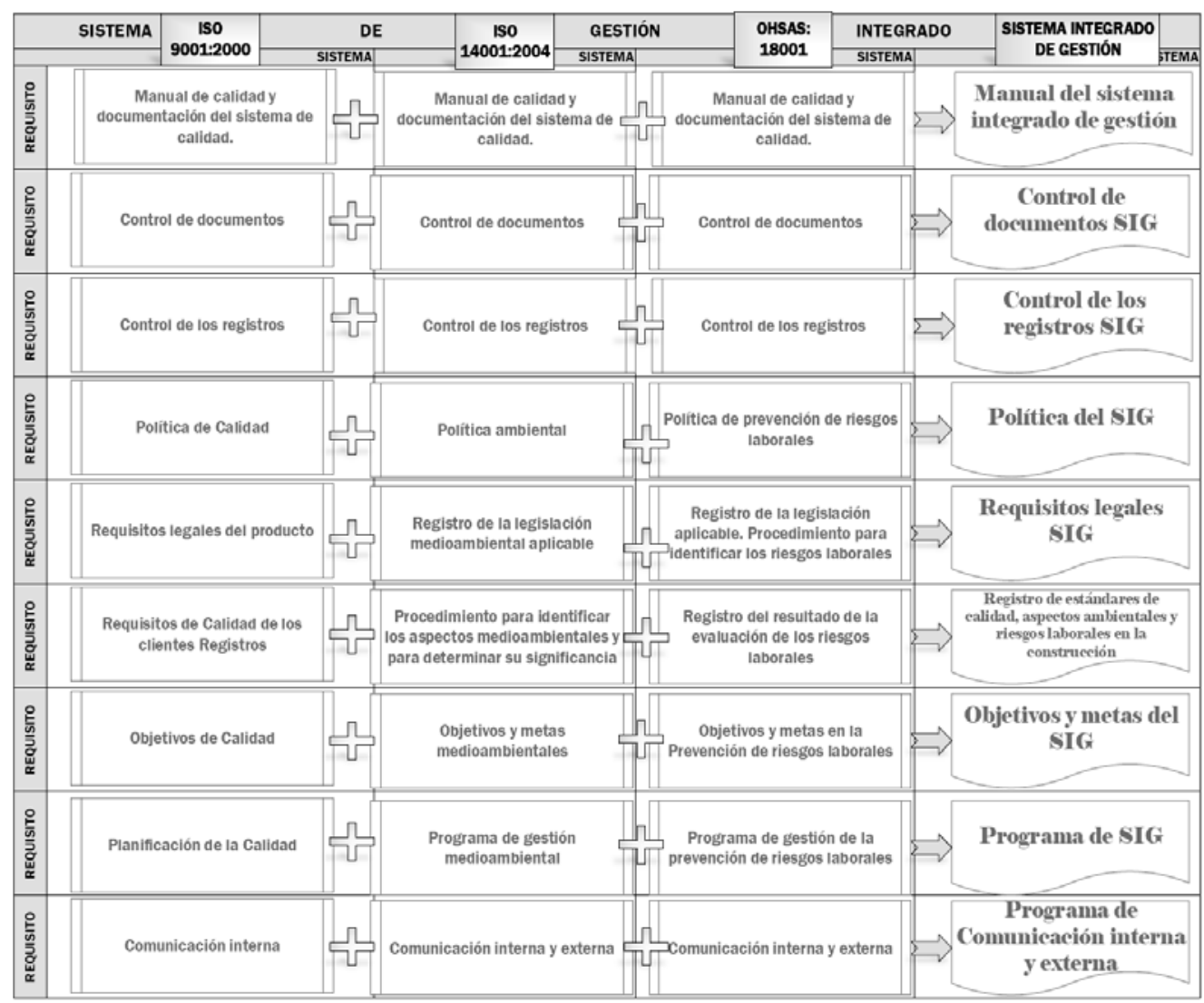

Fuente: Modificación de Fomento, M. D. (2005).

\subsubsection{PRINCIPIOS INTEGRALES DE CONSTRUCCIÓN}

Según lo mencionado en las Norma, I. S. O. (2008), Norma, I. S. O. (2004), OHSAS, N. T. C. (2007), los principios son ejes fundamentales de la ética organizacional y la gestión empresarial, motivo por el que en la presente investigación se presenta 8 directrices que fundamentadas en principios comunes de los 3 sistema de gestión, contribuyen al correcto desempeño del SIG: 


\section{Principios integrales.}

1.- Organización enfocada a los actores claves.- considerados como clientes, trabajadores y sociedad; que se convierten en una finalidad básica. Por ello las organizaciones se integran de diversas formas con las partes interesadas.

2.- Emprendimiento innovador y Liderazgo compartido.- Se desarrolla de la prospección, creatividad y generación de nuevas propuestas dentro de la organización con la implementación del liderazgo compartido de los responsables en las diferentes áreas de la empresa, la dirección de la misma debe crear las condiciones para hacer que la gente participe activamente a través del liderazgo compartido en el logro de los objetivos de la organización.

3.- Participación activa y responsabilidad compartida.- Considerando el involucramiento de la los actores claves a todos los niveles, trabajadores, clientes y sociedad. El talento humano es la esencia de una organización y su involucramiento completo permite el uso de sus competencias, generación de compromiso con el cumplimiento del sistema integral y de su experiencia para el beneficio de la organización.

4.- Gestión integral por procesos (estratégicos, soporte, operativos).- A través de la estandarización de la estructura de la organización, permitiendo la mejora con la optimización de la productividad y efectividad de sus resultados. Orientando la gestión hacia una misión común, compartida y conocida por todas las unidades de la organización, y al mismo tiempo reconoce la interacción de todas las unidades.

5.- Integración de los estándares competitivos a la gestión.- Consiste en la integración de los sistemas de calidad, ambiental salud y seguridad ocupacional, interrelacionando los sistemas para maximizar la eficacia y la eficiencia de la organización.

6.- Capacitación, Comunicación, y retroalimentación.- Estableciendo el desarrollo de tareas, roles, entrenamiento, permanente así como la comunicación interna con los colaboradores del cumplimiento del sistema de gestión integral, colaboración y coordinación en materia de seguridad y salud.

7.-Progreso empresarial continuo.- La mejora permanente de toda la gestión integral, permite la optimización de los procesos en cumplimiento con los objetivos de la organización.

8.- La cultura prevención.- Con la internalización de la cultura de prevención, estableciendo los lineamientos y estándares, para el desarrollo seguro, de calidad y sostenible de la actividad de construcción. Estableciendo los lineamientos, para el desarrollo en un ambiente seguro y saludable. Según el programa de integración 
(Sánchez C. E. A. et al., 2006); menciona que debe realizarse, la adecuación de la legislación vigente, y a la normatividad de tipo voluntario, establecimiento del programa de tareas a realizar, recursos, formación y difusión para dar a conocer el sistema, por ello en la presente investigación se plantean los programas que se describen en los siguientes epígrafes.

\subsection{PROGRAMA DEL SISTEMA DE GESTIÓN INTEGRAL.}

Comprende los siguientes subprogramas:

a) Subprograma de Planificación de la gestión de la calidad. Comprende las siguientes líneas de acción: (control del proyecto, control del proceso constructivo, control de las especificaciones del terreno, adecuada orientación y distribución de espacios.

b) Subprograma de Implementación y Aseguramiento de la gestión de la calidad.-Abarca las siguientes Líneas de acción: (control de los materiales, control de la ejecución, requisitos de durabilidad, integración en su entorno, diseño personalizado ecológicamente sostenible y valorización).

c) Subprograma de control de la Calidad y Flujo del Agua.-Basado en el potencial del impacto ambiental: alteración de la calidad del agua, disminución del recurso hídrico. (Cuba V. A. 2014).

d) Subprograma de protección del Suelo.- Tiene como objetivo la defensa y protección del suelo que serían afectados por las obras que se realizarán, por la probable contaminación por arrojo de residuos líquidos y sólidos. (Cuba V. A. 2014).

e) Subprograma de control de la calidad del aire.- Basado en la probable contaminación por la emisión de material particulado (PM10 y PM2.5) y la contaminación por emisión de gases contaminantes como $\mathrm{CO}_{2}, \mathrm{CO}$ y $\mathrm{SO}_{2}$. (Cuba V. A. 2014).

f) Sub Programa de Manejo de Residuos sólidos.- Tiene como finalidad el cumplir con lo dispuesto en la Ley 27314 (Ley General de Residuos Sólidos y en el D.S. N ${ }^{\circ}$ 057-2004-PCM (Reglamento de la Ley General de Residuos Sólidos). (Cuba V. A. 2014).

g) Sub Programa de Protección del Medio Socio económico.- Fundamentado en el potencial Impacto positivo de generación de puestos de trabajo contribuyendo a un ingreso económico para sus familias.

h) Sub Programa de contratación Temporal de Mano de Obra no Calificada.- Establecido en beneficio del trabajo temporal se hará extensivo al mayor número de jefes de familia empleando el mecanismo de rotación trimestral.

i) Sub Programa de Seguridad y Salud ocupacional.- Líneas de acción comprenden: (capacitación periódica de todo el personal, control médico de salud, inspección de seguridad de los equipos, plan de contingencias de obra, y supervisión de obra. 


\subsection{DE LOS LINEAMIENTOS DE IMPLEMENTACIÓN DEL SISTEMA DE GESTIÓN INTEGRAL}

De acuerdo a la evaluación integral se determinó que la industria de la construcción del Cusco, requiere un instrumento de gestión estructurado, organizado y adaptado al diagnóstico del sector, son los siguientes: (compromiso y responsabilidades compartidas de los actores vinculantes, desarrollo de una planificación consensuada y objetiva, organización en la elaboración del manual de gestión integral participativo, sistematización de procedimientos verificables, documentación de los procedimientos integrales, control operacional de los procedimientos de control integral, monitoreo y verificación del sistema de gestión integral).

\section{CONCLUSIONES Y RECOMENDACIONES}

\section{$\underline{\text { Conclusiones }}$}

- Se planteó, el diseño del sistema de gestión integral, (calidad, ambiental y seguridad - salud ocupacional), para empresas del sector de construcción en la ciudad del Cusco, el cual, permite a las empresas, contar con un plataforma de gestión integral, basado en el diagnóstico, planificación, organización, para una correcta ejecución, supervisión y optimización posterior del sistema.

- El resultado del diagnóstico de la calidad, ambiental, riesgos y salud ocupacional del sector de la construcción en la ciudad del Cusco, permitió identificar los aspectos claves de la gestión integral, mejorando y optimizando los procesos.

- Se presentó el planteamiento de los lineamientos estratégicos de la implementación, del sistema de gestión integral para el sector construcción en la ciudad del Cusco.

\section{$\underline{\text { Recomendaciones }}$}

- El éxito de la implementación del sistema de gestión integral, depende fundamentalmente del grado de compromiso de todos los actores claves, vinculados con la empresa.

- El cumplimiento de los principios del sistema de gestión integral, contribuye a la sinergia, efectividad y optimización de los 3 sistemas.

- La sensibilización, capacitación, comunicación interna y externa del diseño de gestión integral es fundamental, para el cumplimiento preventivo y correctivo de los lineamientos de gestión, el cual deberá contar con una retroalimentación continua horizontal en la empresa. 


\section{REFERENCIAS BIBLIOGRÁFICAS}

ABAD, J., Coll, J., JANE, E., \& Llovera, F. J. (2011), Implicaciones de la integración de los sistemas de gestión de calidad, medio ambiente y seguridad y salud laboral basados en estándares internacionales. Universidad Politécnica de Cataluña. Tesis doctoral, España.

CuBA V. A. (2014), Diseño de un sistema de gestión ambiental basado en la norma ISO 14001:2004, para una empresa constructora. Universidad Internacional Iberoamericana, UNINI. Tesis de Maestría, México.

LEY GENERAL DE RESIDUOS Sólidos 27314, Diario oficial "El Peruano", 21/07/2000. D.S. N 057-2004-PCM (Reglamento de la Ley General de Residuos Sólidos. Diario oficial "El Peruano", 24/04/2004.

Hurtado, F. A. A., Vélez, R. E. B., \& DE los Ríos, J. A. V. (2008), Sistema de gestión integral. Una sola gestión, un solo equipo. Universidad de Antioquia, Gestión y Conocimiento, Colombia,

FOMENTO, M. D. (2005), La gestión por procesos. Modelos para implantar la mejora continua en la gestión de empresas de transporte por carretera. Ministerio de Fomento, España.

NoRMA, I. S. O. (2008), 9001: 2008, Sistema de gestión de la Calidad”, Requisitos, Organización Internacional para la normalización (ISO), Traducción Certificada por el Comité Técnico ISO/TC 176, Gestión de Aseguramiento de la calidad.

NORMA, I. S. O. (2004), 14001: 2004. Sistemas de Gestión Ambiental. Requisitos con orientación para su uso. Traducción certificada. Suiza.

OltRA B., R. F. (2012), Identificación de tendencias de los Sistemas Integrados de Gestión Empresarial. Análisis funcional y diseño de herramientas para el desarrollo de un ERP vertical adaptado al sector cerámico, Editorial Universidad Politécnica de Valencia, Tesis Doctoral, España.

OHSAS, N. T. C. (2007), 18001. Sistema de gestión de la seguridad y salud en el trabajo, AENOR. España.

PARDO, C. I. (2008), Los sistemas y las auditorias de gestión integral. Una herramienta para la mejora y optimización de los procesos y el desempeño en las organizaciones. Universidad de la Salle, Bogotá.

PASTOR, J. R. (2006), Sistema de gestión integrada: calidad, prevención y medio ambiente, Editorial Visión Libros. España.

PARDY, W., \& ANDREWS, T. (2009), Integrated Management Systems: Leading Strategies and Solutions. United States. Government Institutes.

QUINTERO G., M. L. (2013), Gestión sostenible integral: la responsabilidad social empresarial en la integración de los sistemas de gestión, Universidad Politécnica de Valencia, España.

SANTOS, J. R. M. (2004), La gestión integrada: calidad, seguridad y medio ambiente, SERFOREM, SL, México.

SÁnchez, C. E. A., PAlomino, A. E., \& Rivero, J. M. S. (2006), Manual para la integración de sistemas de gestión: calidad, medio ambiente y prevención de riesgos laborales, FC Editorial, Madrid. 